\title{
Die Sicht der Eltern zum altersgemischten Lernen im St. Galler Schulentwicklungsprojekt zu "Schule im alpinen Raum»
}

\section{Robbert Smit und Winfried Humpert}

Eltern sind wichtige Partner bei Schulentwicklungsprojekten, besonders wenn bei einer heterogenen Schülerschaft Massnahmen des altersgemischten Lernens (AdL) und der inneren Differenzierung zur Erhaltung von kleinen alpinen Schulstandorten beabsichtigt werden. Mittels Fragebogen wird die Haltung von 682 Eltern aus 19 Schulen zu Aspekten eines entsprechenden Unterrichts untersucht.

Die inferenzstatistische Datenanalyse erfolgte mit Mittelwertsvergleichen, Pfadmodellen und Mehrebenenanalysen. Es zeigte sich u.a., dass Eltern mit geringerer Leistungsorientierung und einer höheren Zufriedenheit mit der Elternpartizipation eine höhere Zustimmung zum AdL und zum Schulentwicklungsprojekt aufweisen. Die Bedeutung der Befunde für die weitere Entwicklung der alpinen-ländlichen Schulen wird diskutiert.

\section{Einführung}

Kleine Schulen weisen oft ungünstige Schülerzahlen auf, so dass die Klassen nicht immer aufgrund eines Jahrgangs formiert werden können und die Gruppierungen nach andern Kriterien gebildet werden. Zwei bekannte sind Mehrklassenzüge oder altersgemischte Gruppierungen. Während bei Mehrklassenzügen einfach mehrere Jahrgänge unabhängig im gleichen Raum unterrichtet werden, wird beim altersgemischten oder altersdurchmischten Unterricht (AdL) bewusst versucht, die Alters- und Leistungsunterschiede in einer gemeinsamen Lernumgebung einzubetten. Dies erlaubt die Förderung des selbstverantwortlichen Lernens, des Einsatzes älterer Schülerinnen und Schüler als Tutoren der jüngeren (oder leistungsschwächeren) und verringert die «Wettbewerbssituation» untereinander, da die normierte Klasse zu Gunsten vermehrter individualisierter Lernziele aufgebrochen wird. Gefahr besteht in der fehlenden Professionalität der Lehrpersonen im Umgang mit Heterogenität und einem kleineren Lehrkörper mit weniger Fachspezialisten/-innen und Sonderpädagogen/-innen (French, Atkinson \& Rugen, 2007). Insbesondere Eltern wehren sich teilweise 
gegen altersgemischten Unterricht, da er ihnen aus eigener Erfahrung unbekannt ist und ihnen möglicherweise darum Unbehagen bereitet (Nägeli, 2011). Schulentwicklungsprogramme sind bei den Eltern generell oft weniger beliebt als bei den Initianten (Shumow, 2001); darum ist es für Schulen, welche die Einführung von altersgemischten Lerngruppen planen, wichtig, den Eltern passende Informationen anzubieten. Eltern müssen davon überzeugt sein, dass ihre Kinder das notwendige Basiswissen erwerben und der Unterricht auf die Bedürfnisse der Kinder angepasst wird. Fehlt die entsprechende Öffentlichkeitsarbeit, kann dies zu einer Ablehnung von altersgemischten Strukturen in lokalen Abstimmungen führen (Nägeli, 2011).

Das St. Galler Projekt «Adaptive Schulmodelle als Faktor der regionalen Entwicklung» ist Teil des internationalen Forschungs- und Schulentwicklungs-Projekts «Schule im alpinen Raum»(SiaR) und verfolgt Schulentwicklungsmassnahmen zum Umgang mit erhöhter Heterogenität bei Jahrgangs- oder Niveauzusammenlegungen. Wenn im Folgenden vom Projekt SiaR gesprochen wird, ist damit immer das St. Galler Teilprojekt gemeint. Dieses Teilprojekt bestand aus einem Forschungs- und einem zusätzlichen Entwicklungsteil. Schulen konnten sich nur am Forschungsteil oder an beiden Teilen beteiligen. Die Themen der Entwicklung beinhalteten sowohl Elemente auf der Einstellungsebene der Lehrpersonen als auch konkrete methodische Elemente wie kompetenzorientiertes und differenziertes Unterrichten (Keller, 2011). Damit sollten Lehrpersonen für allfällige strukturelle Veränderungen (Zusammenlegen von Jahrgängen oder von Niveaus auf der Oberstufe) vorbereitet werden.

Die im Projekt erhobenen Daten der Elternbefragung erlauben es, Fragen zur Haltung der Eltern zum Projekt und zum altersgemischten Unterricht zu untersuchen. Die von der Pädagogischen Hochschule St. Gallen unterstützten Schulentwicklungsmassnahmen fokussierten auf die Lehrpersonen. Ob und wie die Eltern in strukturelle und unterrichtliche Veränderungsprozesse miteinbezogen wurden, oblag der Initiative der lokalen Schulbehörde oder -leitung. Dies weist auf das bisher vernachlässigte Feld der Elternpartizipation bei Schulentwicklungsprozessen hin.

\section{Altersgemischter Unterricht und Differenzierung}

In altersge- oder altersdurchmischten Klassen (multigrade oder multiage classes) sind Lehrpersonen während eines Schuljahres verantwortlich für den Unterricht von mehreren Alterskohorten (Little, 2001). Gründe dafür können organisatorischer Art sein, wenn bspw. die Schülerzahlen sinken. Es können aber auch pädagogische Überlegungen sein, die zu einem altersdurchmischten Lernen (AdL) führen: Die Vielfalt der Schülerinnen und Schüler wird beim AdL als 
Chance aufgefasst. Pädagogische Vorteile liegen im sozialen und demokratischen Lernen. Ein Pluspunkt wird zudem in der Möglichkeit gesehen, dem Lerngegenstand mehrmalig innerhalb eines mehrjährigen Zyklus zu begegnen und dabei immer den jeweiligen Entwicklungsstand der Schülerinnen und Schüler zu berücksichtigen (Achermann \& Gehrig, 2011). Es wird kindzentriert und kooperativ gelernt, das Lernen wird z.B. durch Portfolios dokumentiert und es erfolgt eine intensive Beratung durch die Lehrperson. Unterrichten in altersgemischten Klassen ist anspruchsvoll und vielen Lehrpersonen fehlt die Erfahrung für einen entsprechenden Unterricht (Little, 2001; Miller, 1991). Die meisten empirischen Untersuchungen zu AdL betreffen die unteren Schulstufen. Bei Miller (1991) findet sich eine Übersicht über den Forschungsstand: Viele Ergebnisse sind neutraler Art, sie zeigen keine oder nur geringe positive Effekte von AdL auf die Schulleistung (Veenmann, 1995). Allerdings ergeben sich auch keine allenfalls befürchteten negativen Effekte. Hattie (2002) merkt zu den geringen Effektgrössen kritisch an, dass die wenigsten Lehrpersonen bei der Umstellung auf AdL ihren Unterrichtsstil ändern.

Altersdurchmischtes Lernen kann einem didaktischen Differenzierungsprinzip folgen, bei dem nicht von der Klassenstufe, sondern vom Leistungsstand jedes einzelnen Schülers und jeder einzelnen Schülerin ausgegangen wird und adaptive Lern- und Lehrformen eingesetzt werden. Nach Miller (1991) sind sechs Schlüsselvariablen für erfolgreiches altersdurchmischtes Unterrichten notwendig:

1. Organisation des Klassenzimmers

2. Klassenführung und Disziplin

3. Unterrichtsstrukturierung und Curriculum

4. Unterrichts-Methoden und Gruppierung der Schülerinnen und Schüler

5. Selbstbestimmtes Lernen und Lernkompetenzen

6. Tutoring durch Peers.

Für die Umsetzung der obigen Punkte lässt sich am besten auf den Differenzierten Unterricht zurückgreifen. Dort wird ebenfalls in kleinen Gruppen, selbstorganisiert und mit Hilfe von Lernpartnern oder der Lehrperson als Coach gelernt. Die Inhalte der Schul- und Unterrichtsentwicklung unseres Projektes fokussierten deshalb Methoden zur Umsetzung von Differenzierung. Für die Eltern dagegen ist ein äusserliches Merkmal oder Ziel des Projektes, wie «altersgemischte Lerngruppe» besser erkennbar.

Bei der «äusseren Differenzierung» werden Klassenverbände oder Lerngruppen (zeitweilig) aufgelöst und nach einem spezifischen Kriterium neu zusammengesetzt (z.B. bei Hochbegabtenklassen oder Sportklassen). Dazu gehört auch die Organisation des Lernens auf der Sekundarstufe in verschiedenen Leistungszügen oder die Aufhebung der Jahrgangszüge beim altersgemischten Lernen. Bei der «inneren Differenzierung» ist hingegen das Ziel, im Anschluss an eine Lern-Diagnose durch die Lehrperson ein differenzierendes 
Lernangebot für unterschiedliche Leistungsgruppen innerhalb der Klasse anzubieten und die Lernenden während der Lernphase optimal zu begleiten (Bräu, 2008; Tomlinson et al., 2003). Hier liegen gleichzeitig auch die Herausforderungen an die Lehrpersonen im differenzierenden Unterrichten mit altersgemischten Lerngruppen begründet.

In einer Literaturübersicht empirischer Arbeiten zur Differenzierung von Tomlinson et al. (2003) wird aufgezeigt, wie Schulentwicklung aufgrund der Befunde zur Differenzierung aussehen könnte. Dabei wird Differenzierung als ein Unterrichtsansatz angesehen, bei dem

...teachers proactively modify curricula, teaching methods, resources, learning activities, and student products to address the diverse needs of individual students and small groups of students to maximize the learning opportunity for each student... (S. 120)

Tomlinson et al. (2003) ziehen aus Metaanalysen empirischer Arbeiten und verschiedenen theoretischen Ansätzen u.a. folgende Schlüsse für erfolgreiche Differenzierung (S. 127 -129):

- Effektive Differenzierung ist vorausplanend und weniger reagierend.

- Effektive Differenzierung erfolgt optimal in kleinen Lehr-Lerngruppen.

- Bei effektiver Differenzierung wird das Lernmaterial variiert.

- Effektive Differenzierung passt das Lerntempo den Bedürfnissen und Fähigkeiten der Lernenden an.

- Effektive Differenzierung basiert auf dem Vorwissen der Lernenden.

Auch für die Praxis der Differenzierung gilt wie beim AdL: Obwohl die meisten Lehrpersonen die Notwendigkeit anerkennen, auf die Heterogenität der Schulkinder im üblichen Klassenzimmer einzugehen, planen oder verwirklichen sie selten Differenzierung im Unterricht (Hattie, 2002). Neuere Forschungen legen jedoch den Nutzen differenzierten Unterrichts nahe. Baumgartner, Lipowski und Rush (2003) konnten z.B. bei Studien zum Lesen-Lernen zeigen, dass differenzierende Anleitungsstrategien die Leseleistung steigern. Einen ähnlichen Befund zur Differenzierung erzielte Tieso (2005) bei Mathematikleistungen auf der Basis von Assessments.

Falls Schulen und Klassen eine verstärkte Differenzierung einführen wollen, müssen die Eltern hierzu gewonnen werden. Dies ist nicht ganz einfach, da Eltern oft Nachteile für ihr Kind befürchten, wenn Unterrichtsentwicklungsmassnahmen eingeführt werden sollen, vor allem wenn die Differenzierung jahrgangsübergreifend ist (French et al., 2007). Gerade für Schulen mit abnehmenden Schülerzahlen kann aber eine (teilweise) Auflösung der Jahrgangsoder Niveauklassen eine Chance zum Erhalt von Schulhäusern in kleinen (alpinen) Ortschaften sein. Zur Implementation von AdL oder innerer Differenzierung im Rahmen der Schulentwicklung ist ein gemeinsamer Entwicklungsweg von Lehrpersonen und Eltern anzustreben. 


\section{Elternpartizipation im Schulentwicklungs- prozess}

Eltern werden als wichtige Partner in einer funktionierenden Schule gesehen. Dabei geht es in erster Linie um die Unterstützung der Kinder zuhause und die Zusammenarbeit mit der Schule bzw. den Lehrpersonen. Elternpartizipation fokussiert auf die Zusammenarbeit und wird von Meister (2010) definiert als Prozess sozialer Teilhabe und interaktiver Einflussnahme an innerschulischen Handlungsabläufen und Entscheidungsprozessen.

Empirische Studien gibt es vor allem zur elterlichen Unterstützung beim Lernen (Epstein \& Dauber, 1991; Hoover-Dempey et al., 2001; HooverDempey et al., 2005; Hoover-Dempey \& Sandler, 1997). Die Ergebnisse zeigen, dass die elterliche Unterstützung bei der Bearbeitung von Hausaufgaben lern- und leistungsförderlich ist. Nebst der Leistung scheint auch das Verhalten der Schülerinnen und Schüler positiv von einem häufigen und guten SchuleEltern-Kontakt beeinflusst zu werden (Izzo, Weissberg, Kasprow \& Fendrich, 1999). Aus Sicht des altersgemischten Lernens, welches von den Lernenden eine grössere Selbständigkeit erwartet, bspw. beim Hilfe-Holen und Hilfe-Geben oder im Umgang mit den unterschiedlichen Leistungsniveaus, ist die Selbstbestimmungstheorie von Deci und Ryan (1985) zur Erklärung von elterlichen Einflüssen auf schulische Leistungen von Belang. Lorenz und Wild (2007) zeigen die Bedeutung intrinsischer Motivation in autonom wählbaren Settings beim Lernen auf. Hohe Leistungsmotivation entwickelt sich dann am besten, wenn Eltern hohe, aber realistische Anforderungen an das Kind stellen (Lorenz \& Wild, 2007; Trautwein, Lüdtke, Schnyder \& Niggli, 2006). Umgekehrt erweisen sich kontrollierende Formen der elterlichen Instruktionen, erfasst als Ausmass leistungsorientierten Drucks durch Eltern, unter motivational-affektiven Gesichtspunkten als abträglich. Eltern mit einem höheren Bildungsabschluss äussern eher ihren Unmut zum Unterricht der Lehrpersonen ihres Kindes (Smith et al., 1997). Allerdings heisst das nicht, dass bildungsnahe Eltern alternativen Schulformen mit altersgemischtem Unterricht generell kritischer gegenüberstehen. Gerade Reformschulen werden häufig bewusst von Eltern aus der akademisch gebildeten oberen Mittelschicht gewählt, weil diese für ihre Kinder ein personales Lernangebot zur Verfügung stellen (Ullrich, 2008).

Zwischen der Elternpartizipation und dem Schulklima scheint eine reziproke Beziehung zu bestehen (Christenson \& Sheridan, 2001). Schulen mit einem positiven, offenen Klima erfreuen sich grösserer Partizipation seitens der Eltern (Griffith, 2000). Das beginnt, so Christenson und Sheridan (2001) mit einer Atmosphäre in der Schule, welche die Eltern willkommen heisst. Dazu gehören weiter eine Schulleitung und eine Schulbehörde, welche offen sind für die Bedürfnisse der Eltern, wie etwa für Schulbusse oder familienergänzende Betreuung. Beide Aspekte sind durchaus von Belang für die Schulen im ländlichen, alpinen Raum, wo die Schulwege oftmals lang sind (Müller, Keller, Kerle, Raggl \& Steiner, 2011). 
Ein wichtiges Feld des Eltern-Schule Kontakts sind Gespräche mit der Klassenlehrperson. Aus Sicht der Eltern ist der direkte Kontakt mit der Lehrperson ihres Kindes nach wie vor der wichtigste Aspekt von Elternpartizipation (Shumow, 2001). Aufschlussreich dabei ist, dass Eltern in Shumows Untersuchung bei Lehrer-Eltern-Gesprächen mehr Interesse am Wohlbefinden ihres Kindes als an den Ergebnissen in Prüfungen zeigten. Aich und Behr (2010) machen auf fehlende sozial-kommunikative Kompetenzen bei Lehrpersonen aufmerksam, was bei Lehrpersonen dazu führen kann, Eltern-Lehrer-Gespräche zu vermeiden. Auf Seiten der Lehrpersonen werden viele Elterngespräche als problembehaftet und belastend erlebt. Die Beziehung zwischen Lehrperson und Eltern ist in der Realität von einem Ungleichgewicht geprägt. Die Lehrperson sieht sich als Fachperson und nimmt eine oft eher bevormundende Rolle ein, während sich die Eltern sehr oft zurücknehmen, da sie ja schliesslich nicht die Experten sind (Shumow, 2001). Entsprechend bräuchte es auch für Eltern entsprechende Trainings (Wild, 2003).

Der bisherige Einsatz der Eltern in der Schule wird eher kritisch gesehen, wie ein Aufsatz von Klaffke (2000) bereits vor über einem Jahrzehnt deutlich macht:

Elternarbeit in Schulen beschränkt sich oft auf Hilfstätigkeiten wie beispielsweise Kuchenverkauf bei Festen. Viele Schulen versuchen in einer Art «Vermeidungshaltung» zu verhindern, dass Eltern «einen Blick hinter die Kulissen werfen». Hospitation oder Mitarbeit von Eltern im Unterricht sind geradezu unerwünscht. (Klaffke, 2000, S. 16)

Kliewe, Schreiber und Wiganek (2006) machen auf die Bedeutung der Schaffung von Gelegenheitsstrukturen für den Schule-Elternkontakt aufmerksam, wenn Eltern und Schule einander wertschätzende langfristige Kooperationspartner werden sollen. Neben gesetzlichen Grundlagen der Eltern-Schule-Kooperationsformen und behördlich unterstützten Fördermassnahmen gehören zu den festen Kooperationsformen auch niederschwellige Angebote wie Hausbesuche der Lehrpersonen. Das Engagement innerhalb der Elternpartizipation ist abhängig vom sozio-ökonomischen Status der Eltern (Simon, 2004). Insofern sind auch Formen zu finden, welche etwa Eltern mit einem Migrations-Hintergrund ansprechen.

Während die häusliche Mitarbeit der Eltern eine unbestrittene Bedeutung für den Leistungsfortschritt von Schülerinnen und Schülern hat und an vielen Orten die gesetzlich vorgeschriebenen Elternvertretungen realisiert sind, ist die Mitarbeit von Eltern bei pädagogischen Fragen oder im Rahmen der Schulentwicklung weitestgehend unbekannt. Die gesetzlichen Möglichkeiten in den Schweizer Kantonen sind beschränkt, am häufigsten dürfen Eltern bei der Entwicklung eines Betreuungsangebots mitentscheiden (Cusin \& Grossenbacher, 2001). In der Forschungsliteratur zur Schulentwicklung tauchen Eltern auch eher wenig auf (Langer, 2011). In letzter Zeit wird aber in der 
Bildungspolitik, der Erziehungswissenschaft wie in der Öffentlichkeit das Thema Elternmitarbeit bei Schulentwicklungsfragen verstärkt diskutiert.

Empirische Studien zur Sicht der Eltern hinsichtlich der Teilnahme an der Schulentwicklung sind wenig ermutigend. "Eltern beklagen unzureichende Mitbestimmungsmöglichkeiten und Informationsdefizite...» (Meister, 2010, S. 277). Die aktuelle Schulmanagement-Diskussion weist den Eltern zudem eine geringe Bedeutung bei der Schulentwicklung zu (Meister, 2010, S. 278). Auch im Rahmen von Studien zur Schulprogrammentwicklung scheint die Mitarbeit der Eltern über längere Zeit nur für einen kleinen Teil der Eltern attraktiv zu sein (Arnold et al., 2004). Nicht alle Eltern müssen bei Schulentwicklungsprozessen mitmachen; es ist sinnvoll, mit Fokusgruppen zu arbeiten. Allerdings ist darauf zu achten, Eltern aus allen sozialen Milieus miteinzubeziehen. Gemäss Langer (2011) halten sich bildungsferne Eltern, weil kapitalarm und wenig organisationsfähig, aus der Partizipation an Schulentwicklung heraus.

Schulentwicklungsprozesse - und damit eingeschlossen Unterrichtsentwicklung - können top-down oder bottom-up eingeleitet werden. Oftmals besteht jedoch einfach ein mehr oder weniger drängendes Problem oder ein Veränderungswunsch als Ausgangspunkt von Schulentwicklungsarbeit (Gauz, 2000; Sommer-Sutter in dieser Nummer). Initianten können Akteure innerhalb der Schule selbst (Lehrpersonen, Schülerinnen und Schüler, Schulleitung), mit der Schule Verbundene (Schulbehörde, Eltern) oder auch Aussenstehende (bspw. aus der Politik, Wirtschaft) sein. Der Einbezug der Eltern ist hilfreich bei der Bestandsaufnahme und der Zukunftsperspektive einer lokalen Schule. Eltern sind ein unabdingbarer Feedbackgeber. In einem Brandenburger Schulprojekt wurde nebst einem Elternfeedback zusätzlich gefragt, welche konkrete Unterstützung denn Eltern anbieten würden (Kliewe et al., 2006). Eltern können aber auch bei der Interpretation und Diskussion der Ergebnisse wertvolle Hilfe leisten. Eltern sollen zudem bei Veränderungsprozessen informiert, wenn nicht gar bei der Umsetzung miteinbezogen werden. Projekte haben dann Erfolg, wenn deren Inhalte zu einem gemeinsamen Anliegen von Schulleitung, Lehrpersonen, Schülerinnen und Schülern und eben Eltern geworden sind. Hilfreich für die Akzeptanz könnte auch eine Übergangszeit sein, in welcher sich betroffene Schülerinnen und Schüler mit ihren Eltern an die neuen Strukturen anpassen können. 


\section{Forschungsfragen}

Aufgrund der bisherigen Darstellungen ergeben sich folgende Forschungsfragen:

- Wie stehen Eltern zum altersgemischten Unterricht?

- Was fördert eine positive Haltung zum altersgemischten Unterricht?

- Wie stehen Eltern zum Schulentwicklungsprojekt Schule alpin?

- Welche Bedeutung hat die Elternpartizipation für die Akzeptanz des Schulentwicklungsprojekts?

\section{Methode}

\section{Stichprobe}

Am Forschungsprojekt teilgenommen haben Schulen aus dem ländlich-alpinen Raum des Kantons St. Gallen und bei der Primarstufe auch eine Schule aus Liechtenstein sowie zwei Schulen aus dem Kanton Glarus. Zusätzlich in der Stichprobe befinden sich zwei Privatschulen mit individualisierendem Unterricht als Vergleichsgruppe. Die einzelnen Teilstichproben sind aus der Tabelle 1 ersichtlich. Lehrpersonen und Schülerinnen und Schüler wurden zweimal (Herbst 2009) und (Frühjahr 2011) befragt. Die Eltern wurden lediglich einmal befragt.

Der Rücklauf bei den Fragebogen liegt für Lehrpersonen und Schülerinnen und Schüler bei rund 80\%. Bei den Eltern hat zum Zeitpunkt Frühjahr 2011 knapp die Hälfte mitgemacht.

\section{Tabelle 1: Stichprobe der Eltern}

\begin{tabular}{lccccccccc}
\hline & \multicolumn{2}{c}{ Schulen } & \multicolumn{2}{c}{ Schultyp } & \multicolumn{2}{c}{ Geschlecht } & \multicolumn{2}{c}{ Familiensprache } \\
& Primar & $\begin{array}{c}\text { Sekun- } \\
\text { dar }\end{array}$ & Primar & $\begin{array}{c}\text { Sekun- } \\
\text { dar }\end{array}$ & $\begin{array}{c}\text { weib- } \\
\text { lich }\end{array}$ & $\begin{array}{c}\text { männ- } \\
\text { lich }\end{array}$ & & $\begin{array}{c}\text { deutsch } \\
\text { fremd- } \\
\text { sprachig }\end{array}$ \\
\hline ohne Schulentwicklung & 2 & 4 & 11 & 141 & 104 & 48 & 138 & 14 \\
mit Schulentwicklung & 5 & 6 & 65 & 417 & 351 & 117 & 442 & 32 \\
$\begin{array}{l}\text { Privatschulen mit } \\
\text { Individualisierung }\end{array}$ & 0 & 2 & 0 & 48 & 34 & 13 & 37 & 7 \\
Total & 7 & 12 & 76 & 606 & $489^{*}$ & $178^{*}$ & $617^{*}$ & $53^{*}$ \\
\hline
\end{tabular}

*fehlende Werte nicht aufgeführt

\section{Design}

Das Schulentwicklungsprojekt dauerte von Sommer 2009 bis Ende 2011, wobei die reine Forschungsphase ein halbes Jahr früher endete. Eine Übersicht über die eingesetzten Instrumente findet sich in Abbildung 1. Auf der Oberstufe und bei den erwachsenen Akteuren wurde zumeist ein Online-Fragebogen eingesetzt. 
Für die Primarschülerinnen und -schüler fand am häufigsten die Papierversion Verwendung. Anschliessend an die erste Erhebung startete die Schulentwicklung.

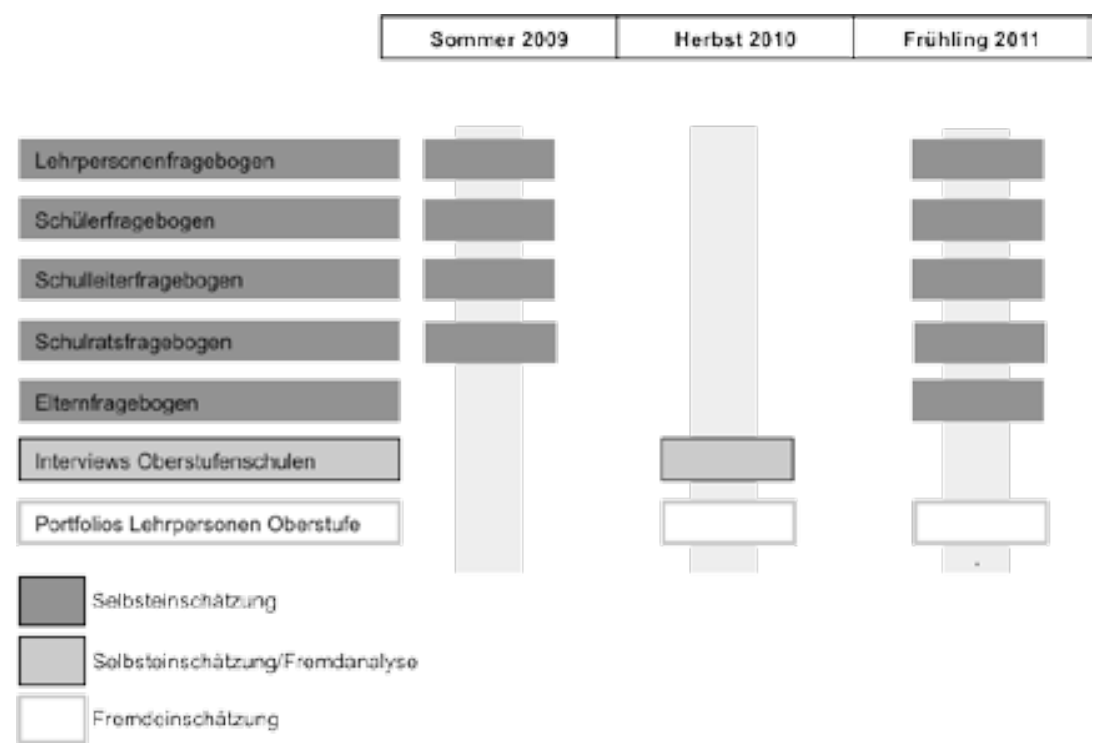

Abbildung 1: Forschungsdesign des St. Galler Teilprojekts von Schule alpin

Eine gewisse Problematik des Forschungsdesigns besteht darin, dass nur schon die Teilnahme am Schulentwicklungsprojekt eine stärkere Sensibilisierung der Akteure darstellt und die Elternmeinung beeinflusst. Inwiefern bei dieser Untersuchung die Eltern betroffen sind, lässt sich anhand der Daten nicht klären. Der Hawthorne-Effekt wurde dadurch gemildert, dass alle Schulen jeweils nach der ersten Befragung eine Rückmeldung erhielten, welche es auch den nicht am Schulentwicklungsprojekt beteiligten Schulen ermöglichte, eine Standortbestimmung vorzunehmen und diese mit den Eltern zu besprechen.

\section{Instrumente}

Es wurde ein Fragebogen auf der Basis der klassischen Testtheorie entwickelt. Jeweils ein Elternteil der befragten Schülerinnen und Schüler (Gesamtstichprobe $\mathrm{n}=682$ ) füllte einen Fragebogen aus, der Items zu acht Skalen enthielt (Tabelle 2). Zusätzlich wurden der Bildungshintergrund (höchster Schulabschluss) und die Häufigkeit der Hilfe bei den Hausaufgaben erhoben. Für die Schüler- und Lehrpersoneninstrumente siehe Smit, Humpert, Obertüfer-Gahler, Engeli und Breuer-Brodmüller (2011). Zu allen Skalen fand eine Überprüfung der Reliabilität statt (Cronbach $\alpha>$.70). 
Tabelle 2: Skalen des Elternfragebogens

\begin{tabular}{lclcc}
\hline Elternskalen & $\begin{array}{c}\text { Anzahl } \\
\text { Items }\end{array}$ & Beispielitem & M & SD \\
\hline $\begin{array}{l}\text { Leistungsorientierung } \\
\text { (-druck) }\end{array}$ & 4 & $\begin{array}{l}\text { Ich möchte, dass unsere Tochter/unser } \\
\text { Schulkn zuhause zusätzlich ïbt. }\end{array}$ & 3.28 & .53 \\
Klassenklima & 4 & $\begin{array}{l}\text { An der Schule gibt es klare Regeln, wie die } \\
\text { Schüler sich zu verhalten haben. }\end{array}$ & 3.43 & .45 \\
Elternpartizipation & 3 & $\begin{array}{l}\text { Die Schüler/innen haben es gut in ihrer } \\
\text { Klasse. }\end{array}$ & 3.16 & .46 \\
$\begin{array}{l}\text { Unterrichtssituation } \\
\text { (Adaption und Engagement) }\end{array}$ & 4 & $\begin{array}{l}\text { Die Eltern können sich in der Schule } \\
\text { genügend einbringen und mitwirken. } \\
\text { Die Leistungsanforderungen sind passend. }\end{array}$ & 3.14 & .43 \\
$\begin{array}{l}\text { Haltung zur Differenzierung } \\
\text { Haltung zu altersdurch- }\end{array}$ & 5 & $\begin{array}{l}\text { Die Berücksichtigung der individuellen } \\
\text { Möglichkeiten eines Schülers ist mir... } \\
\text { mischtem Lernen (AdL) }\end{array}$ & 4.16 & .55 \\
$\begin{array}{l}\text { Einstellung zum Projekt } \\
\text { Schule alpin* }\end{array}$ & 4 & $\begin{array}{l}\text { Gemeinsames Lernen in altersgemischten } \\
\text { Gruppen kann ich mir gut vorstellen. } \\
\text { Die Ziele des Projekts verbessern die } \\
\text { Situation der Schule im Dorf. }\end{array}$ & 2.99 & .49 \\
\hline Ant & 2.88 & .76 \\
\hline
\end{tabular}

Anmerkungen: $\mathrm{n}=677-682$; $\mathrm{n}=281$; Skala 1-4 ( 4 = hoch), ausser Haltung Differenzierung 1-5

\section{Datenanalyse}

Die quantitativen Auswertungen wurden mithilfe der Statistikprogramme PASW, Mlwin und Mplus durchgeführt. Dabei wurden zunächst deskriptive Analysen (Häufigkeiten, Korrelationen) und anschliessend inferenzstatistische Verfahren (Mittelwertsvergleiche, Regressionsanalysen, Strukturgleichungsmodelle) angewendet. Obwohl Schulen alle Eltern gleich behandeln, nehmen Eltern das Angebot der Schulen doch individuell wahr. Zudem sind auch nicht alle Eltern gleich betroffen oder engagiert bei Schule-Eltern-Problemen. Aus diesen Gründen sind, wo es die Stichprobe ermöglicht, sowohl die Varianz innerhalb der Schulen (resp. Schulorte) und zwischen den Schulen zu berücksichtigen (Mehrebenenmodelle).

\section{Ergebnisse}

\section{Zusammenhänge der Elternskalen}

Zunächst werden die Zusammenhänge zwischen den verschiedenen Skalen dargestellt (siehe Tabelle 3).

Die höchsten signifikanten Zusammenhänge zeigen sich zwischen der Variablen «Unterrichtssituation» (passende Anforderungen und herrschende Arbeitshaltung) und den Dimensionen "Klassenklima» ( $r=0.76)$ oder «Schulklima» $(r=0.54)$, welche selber wiederum stark korrelieren. 


\begin{tabular}{|c|c|c|c|c|c|c|c|c|}
\hline & 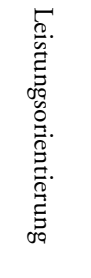 & 呑 & 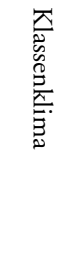 & 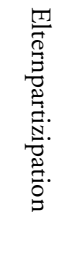 & 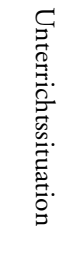 & 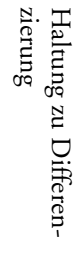 & 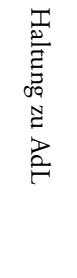 & 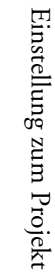 \\
\hline Leistungsorientierung & 1 & & & & & & & \\
\hline Schulklima & .03 & 1 & & & & & & \\
\hline Klassenklima & .04 & $.60^{* *}$ & 1 & & & & & \\
\hline Elternpartizipation & -.06 & $.53^{* *}$ & $.40^{* *}$ & 1 & & & & \\
\hline Unterrichtssituation & .06 & $.54^{* *}$ & $.76^{* *}$ & $.49^{* *}$ & 1 & & & \\
\hline Haltung zu Differenzierung & $.12^{* *}$ & $.22^{* *}$ & $.19^{* *}$ & $.25^{* *}$ & $.26^{* *}$ & 1 & & \\
\hline Haltung zu AdL & $-.15^{* *}$ & $.24^{* *}$ & $.25^{* *}$ & $.30^{* *}$ & $.34^{* *}$ & $.13^{*}$ & 1 & \\
\hline Einstellung zum Projekt ${ }^{\circ}$ & $.12^{*}$ & $.23^{* *}$ & $.22^{* *}$ & $.26^{* *}$ & $.30^{* *}$ & $.11^{*}$ & $.32^{* *}$ & 1 \\
\hline
\end{tabular}

Anmerkung: $\mathrm{n}=677-682,{ }^{\circ} \mathrm{n}=281 ;{ }^{* *} \mathrm{p}<.05,{ }^{*} \mathrm{p}<.01$.

Mit andern Worten: Je angemessener die Arbeitsanforderungen gesehen werden und je grösser das Engagement der Schülerinnen und Schüler aus Sicht der Eltern ist, desto besser ist auch das Klassenklima bzw. Schulklima und umgekehrt. Eine gelebte Elternpartizipation ist Teil eines guten Schulklimas $(r=.53)$. Partizipation und Zufriedenheit mit der Unterrichtssituation weisen ebenfalls einen mittelhohen Zusammenhang auf. Die Haltung zum altersdurchmischten Lernen entspricht der Haltung zum Projekt Schule alpin. Je positiver die Einstellung zum Projekt Schule alpin ist, desto positiver ist auch die Haltung zum AdL und umgekehrt $(r=.32)$.

Erstaunlicherweise haben Eltern mit einer positiven Haltung zur Differenzierung nicht automatisch eine gleich positive Haltung zum AdL, welches ebenfalls vermehrt individuelle Lernziele beinhaltet. Die Korrelation ist sehr gering $(r=.13)$, wenngleich aufgrund des grossen $n$ eine Signifikanz vorliegt.

Von Interesse ist natürlich auch ein Vergleich der Sicht der Eltern, deren Kinder sich in Klassen befanden, die an der Schulentwicklung teilnahmen bzw. nicht teilnahmen (siehe Tab. 4). 
Tabelle 4: Varianzanalyse zur Überprüfung von Unterschieden zwischen den Gruppen ohne Schulentwicklung (SE), mit Schulentwicklung (SE) und Privatschulen

\begin{tabular}{lcccccccc}
\hline & \multicolumn{2}{c}{ ohne SE } & \multicolumn{2}{c}{ mit SE } & \multicolumn{2}{c}{ Privatschulen } & & \\
& \multicolumn{2}{c}{$n=152$} & \multicolumn{2}{c}{$n=480$} & $n=48$ & & \\
& $M$ & $S D$ & $M$ & $S D$ & $M$ & $S D$ & $F$ & $\eta^{2}$ \\
\hline Leistungsorientierung & 3.30 & .52 & 3.29 & .54 & 3.22 & .51 & .45 & .00 \\
Schulklima & 3.33 & .45 & 3.46 & .44 & 3.39 & .48 & $5.41^{*}$ & .02 \\
Klassenklima & 3.12 & .42 & 3.18 & .47 & 3.17 & .49 & .93 & .00 \\
Partizipation & 3.31 & .56 & 3.33 & .59 & 3.35 & .60 & .11 & .00 \\
Unterrichtssituation & 3.07 & .37 & 3.16 & .43 & 3.15 & .52 & 2.59 & .01 \\
Haltung zu Differenzierung & \\
Haltung zu AdL & 4.13 & .58 & 4.16 & .54 & 4.19 & .58 & .33 & .00 \\
& 2.87 & .54 & 3.00 & .47 & 3.21 & .40 & $9.38^{*}$ & .03 \\
\hline
\end{tabular}

Anmerkung: Skala $1-4,4$ (trifft genau zu), ${ }^{\circ}$ Skala $1-5 .{ }^{*} p<.05$

Aus der Tabelle 4 wird ersichtlich, dass die Skalenwerte recht hoch liegen, die Skala «Haltung zu Differenzierung» ist fünfstufig. Die Skala «Haltung zum altersgemischten Unterricht» (AdL) weist die tiefsten Zustimmungswerte auf. Hier zeigen sich aber auch die grössten Unterschiede zwischen den drei Gruppen. Beim Schulklima zeigen sich ebenfalls geringe, aber signifikante Mittelwertsunterschiede zwischen den Gruppen mit und ohne Schulentwicklung. Die Effektstärken sind jedoch jeweils äusserst gering. In der Tendenz urteilen die Eltern, deren Kinder in Klassen mit Schulentwicklung sind, positiver als Eltern, deren Kinder sich in Klassen ohne Schulentwicklung befinden. Die Privatschulen mit individualisiertem Unterricht weisen im Vergleich hohe Zustimmungswerte im Bereich «Haltung zu Differenzierung» und «AdL» auf, ansonsten zeigen sich keine Unterschiede.

\section{Haltung der Eltern zum altersgemischten Lernen}

Zur Prüfung, ob sich die Haltung der Eltern zum altersgemischten Unterricht in den verschiedenen Schulen unterscheidet, wurden die Varianzanteile der Variablen innerhalb der Schulorte und zwischen den Schulorten berechnet. Rund $15 \%$ der Varianz liegt dabei zwischen den Orten. Der grösste Teil der Varianz (85\%) befindet sich aber innerhalb der Orte. Die Eltern in den Orten unterscheiden sich bezüglich ihrer Haltung zum altersgemischten Unterricht signifikant, dies zeigt sich in Abbildung 2. Die sechs Orte mit den niedrigsten Werten liegen unter Berücksichtigung der Konfidenzintervalle unter den Werten der vier Orte mit den höchsten Werten. Deutlich ist auch zu sehen, dass die Eltern der an der Schulentwicklung teilnehmenden Schulorte gesamthaft eine positivere Einstellung zum altersgemischten Unterricht aufweisen. Die Privatschulen liegen an dritter und siebter Stelle. 


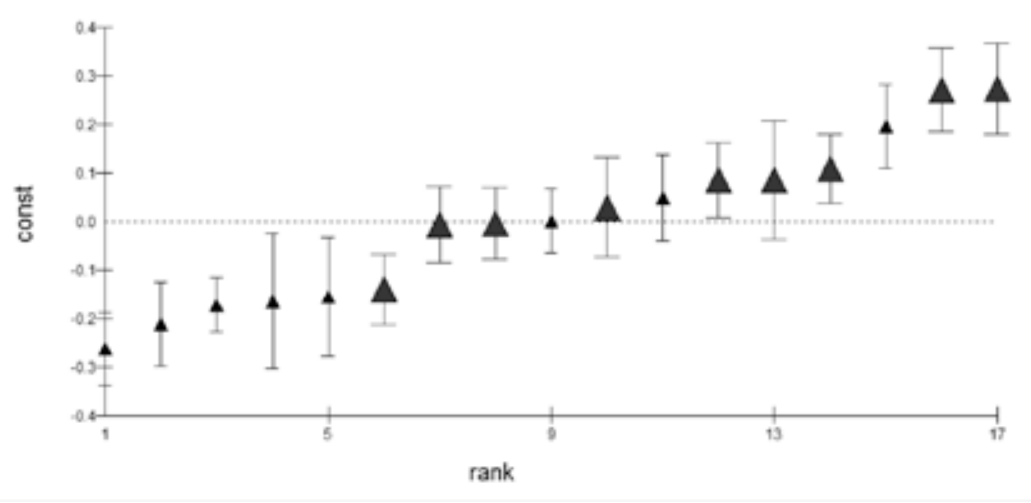

Abbildung 2: Residuen der Schulorte zur Haltung zu AdL mit Konfidenzintervall (grosses Dreieck $=$ Schulen mit Schulentwicklung)

Mittels einer Mehrebenenanalyse wurde geprüft, welche Variablen sich zur Aufklärung der Residualvarianz eignen. Präsentiert wird hier das finale Modell mit der höchsten Aufklärungskraft (siehe Tabelle 5). Die Hintergrundvariablen "Primarschule», "Nationalität» oder «Schulabschluss der Eltern» klären bei diesem Modell keine Varianz auf, die Beta-Werte sind nicht signifikant. Dabei lässt sich für das Modell zur Vorhersage der Haltung der Eltern zum altersgemischten Unterricht in den verschiedenen Schulorten der Anteil der erklärten Varianz nach einer Formel von Bryk und Raudenbush (1992, zitiert nach Hox, 2002, S. 64f.) bestimmen. Um den Wert der erklärten Varianz $\left(R^{2}\right)$ zu bestimmen, wird für jede Ebene getrennt die Reduktion der Varianz für das jeweilige Modell als Anteil an der Gesamtvarianz des «empty»-Modells (ohne Prädiktoren) berechnet.

Eine Veränderung der Varianz zeigt sich auf der Schul- und Elternebene. Diese Effekte sind auf die Prädiktoren «Leistungsorientierung», «Elternpartizipation» und «Unterrichtssituation» zurückzuführen. Die drei Prädiktoren klären 57\% der Varianz auf der Schulebene und 11\% auf der Individualebene auf. Eltern mit einer geringeren Leistungsorientierung und einer höheren Zufriedenheit mit der Elternpartizipation und der Unterrichtssituation weisen eine höhere Zustimmung zum altersgemischten Unterricht auf. Dies gilt unabhängig von der Schulstufe, der Nationalität und des Schulabschlusses der Eltern. 
Tabelle 5: Mehrebenenmodell zur Vorhersage der Haltung der Eltern zum AdL-Unterricht

\begin{tabular}{lcc}
\hline & Haltung AdL & \\
\hline & Schätzwert $\beta$ & s.e. \\
intercept & 3.04 & .04 \\
Fixe Effekte & & \\
Eltern-Ebene & & \\
Leistungsorientierung & $-.11^{*}$ & .03 \\
Schul-Ebene & & \\
Elternpartizipation & $.14^{*}$ & .03 \\
Unterrichtssituation & $.26^{*}$ & .05 \\
$R^{2}$ Schule & .57 & \\
$R^{2}$ Eltern & .11 & \\
\hline
\end{tabular}

Anmerkung: $n=672 ;{ }^{*} p<.05$, s.e.: Standardfehler.

Haltung zum Schulentwicklungsprojekt

Für die an der Schulentwicklung Beteiligten ist es von Belang, die Frage der Unterstützung seitens der Eltern zu klären. Da die Zahl der an der Schulentwicklung beteiligten Schulorte etwas klein ist für Analysen auf mehreren Ebenen, beschränken sich die folgenden Regressionsanalysen auf die Ebene der Eltern. Wie in Tabelle 6 ersichtlich, ist es primär die Haltung der Eltern zum altersgemischten Lernen, welche den Grad der Zustimmung zum Schulentwicklungsprojekt bestimmt.

Tabelle 6: Regressionsmodell zur Vorhersage der Haltung der Eltern zum Schulentwicklungsprojekt

\begin{tabular}{lccc}
\hline Prädiktoren & B & s.e. & $\beta$ \\
\hline Höchster Schulabschluss & -.16 & .05 & $-.19^{* *}$ \\
Leistungsorientierung & .25 & .07 & $.19^{* *}$ \\
Elternpartizipation & .21 & .08 & $.16^{* *}$ \\
Haltung zu altersgemischtem & .48 & .10 & $.30^{* *}$ \\
Lernen & & & \\
\hline
\end{tabular}

Anmerkung: Korr. $R^{2}=.19 ; F(4,279)=17.05 ; p<.01$.

Zudem sind Eltern mit einem weniger hohen Schulabschluss und einer hohen Leistungsorientierung dem Projekt gegenüber positiver eingestellt. Auch eine positiv bewertete Elternpartizipation fördert die Zustimmung zum Projekt. Gesamthaft kann damit aber nur 19\% der Varianz zwischen den Elternmeinungen zum Schulentwicklungsprojekt erklärt werden. 


\section{Elternpartizipation und Innovationsbereitschaft des Teams}

Wie sich in den beiden vorgehenden Analysen gezeigt hat, ist die Elternpartizipation sowohl bei der Einstellung der Eltern zum altersgemischten Unterricht wie auch bei der Haltung zum Schulentwicklungsprojekt von Bedeutung. Hier könnten Lehrpersonen oder Teams ansetzen, wenn sie die Zustimmung der Eltern zu den Zielen des Projektes vergrössern möchten. Anhand der Daten der Lehrpersonen lässt sich prüfen, ob bestimmte Merkmale seitens der Lehrpersonen zu einer guten Elternpartizipation führen. Ordnet man die Lehrpersonendaten über die Schüler den Eltern zu, können diese als Prädiktoren für die Zufriedenheit der Eltern mit der Partizipation dienen. Dies war für einen Teil der Daten $(n=115)$ möglich. Dabei stammen die Daten aus allen drei Gruppen (mit/ohne SE, Privatschulen). Bezüglich der Einschätzung der Elternpartizipation unterscheiden sich die Eltern in den verschiedenen Orten. Für den Gesamtdatensatz liegen rund $10 \%$ der Gesamtvarianz zur Elternpartizipation zwischen den Orten. Nimmt man jedoch nur den Teildatensatz $(n=$ 115), zeigen sich keine Unterschiede zwischen den Orten mehr und es lassen sich nur Aussagen zur Elternebene machen. Von Bedeutung zur Aufklärung der Elternvarianz erweist sich nur die Einschätzung der Lehrpersonen zur Innovationsbereitschaft ihres Teams mit einem $\beta$ von .24 $(\mathrm{F}(1,113)=6.73 ; p<0.05)$. Lehrpersonen, welche ihr Team als innovationsbereit einschätzen, scheinen bei konkreten Umsetzungen der Innovationen auch die Eltern mit einzubeziehen oder zumindest zu informieren. Gefragt wurde bei der Innovationsbereitschaft, ob das Team in der Lage ist, pädagogische Ideen auch praktisch umzusetzen, ob die fachlichen Ressourcen der einzelnen Lehrpersonen im Team optimal eingesetzt werden und ob Ideen und Anregungen vom Team konstruktiv aufgenommen werden. Interessant ist, dass drei weitere Aspekte der Teamkultur Teamklima, pädagogische Grundorientierung, pädagogische Konferenzkultur - keine Rolle bei der Haltung zum Projekt spielen. Man kann also nicht generell von einem positiven Einfluss einer guten Teamkultur auf die Elternpartizipation schliessen.

\section{Pfadmodell zur Haltung der Eltern zu AdL und zum Projekt SiaR}

Die Bewertung des Unterrichts hängt, gemäss Shumow (2001), zu einem grossen Teil von der Rückmeldung der eigenen Kinder ab. Für rund 150 Eltern unserer Stichprobe konnten die Daten der Kinder zugeordnet werden, dabei zeigen sich schwache signifikante Korrelationen einer positiven Einschätzung des Unterrichts seitens der Eltern mit der Höhe der Einschätzung der Diagnosekompetenz der Lehrperson und des Schulklimas seitens der Schüler und Schülerinnen.

Nimmt man die beiden Regressionsmodelle zu altersgemischtem Lernen (Tab. 5) und Einstellung zum Projekt (Tab. 6) zusammen, ergibt sich das Pfadmodell in Abbildung 3. Eine Modellverbesserung fand durch die Integration der Skala 
«Einstellung zur Differenzierung» statt. Es wird davon ausgegangen, dass die Haltung zur Differenzierung einen Einfluss auf die Wahrnehmung der Unterrichtssituation aufweist (Shumow, 2001).

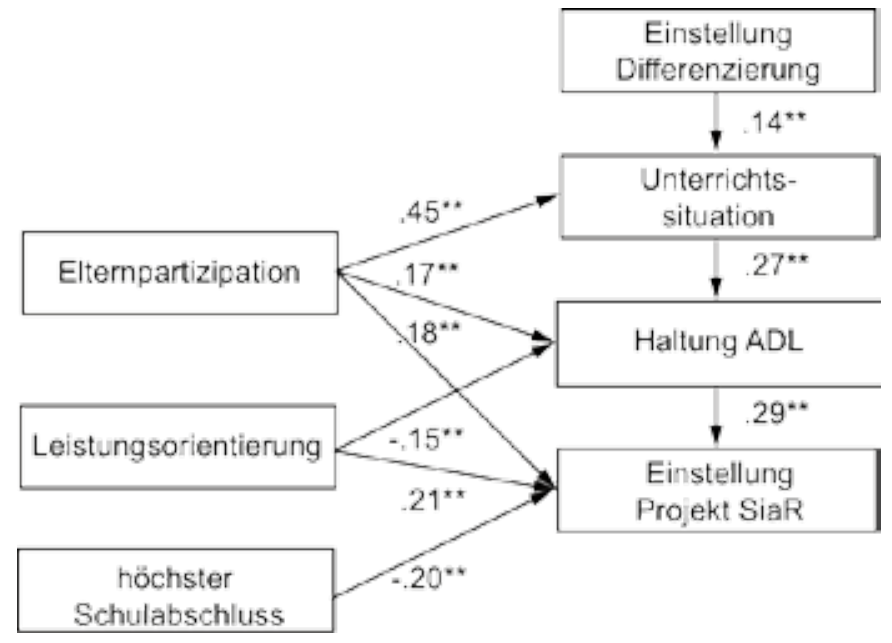

Abbildung 3: Pfadmodell zur Erklärung der Elternhaltung zu AdL und zum Projekt Schulen im alpinen Raum (SiaR); standardisierte Beta-Werte, ${ }^{* *} \mathrm{p}<.01$

Das Modell basiert auf dem Gesamtdatensatz und weist gute Fit-Werte auf: $N=671, \mathrm{Chi}^{2}=14.95, \mathrm{df}=6, p=.02, \mathrm{CFI}=.98, \mathrm{TLI}=.94, \mathrm{RMSEA}=$ .05 . Fehlende Werte wurden mit der Full Information Maximum Likelihood (FIML)-Methode als Schätzwerte ins Modell miteinbezogen (Lüdtke, Robitzsch, Trautwein \& Köller, 2007). Zur Varianzaufklärung $\left(R^{2}\right)$ der Haltung der Eltern zum altersgemischten Lernen trägt das Modell $17.5 \%$ bei, für die Einstellung zum Projekt 21.2\% und zur Einschätzung der Unterrichtssituation 26.1\%. Was in den vorgehenden Analysen noch nicht zur Geltung kam, ist der relativ hohe Einfluss der Elternpartizipation auf die Zufriedenheit mit dem Unterricht (passende Anforderungen und gutes Arbeits- und Lernverhalten) mit einem $\beta$ von .45, welcher wiederum einen deutlichen Einfluss auf die Haltung zum altersgemischten Unterricht besitzt. Damit zeigt sich ein im Vergleich zum direkten Einfluss relativ hoher indirekter Einfluss der Elternpartizipation auf die Haltung zum altersgemischten Unterricht. Das Gleiche lässt sich auch für die Einstellung zum Projekt Schule alpin (resp. deren St. Galler Teil) sagen. Auch hier ist der indirekte Einfluss der Elternpartizipation deutlich höher als der direkte Einfluss, womit sich ihre Bedeutung nochmals verstärkt manifestiert. 


\section{Diskussion}

Ohne Einbezug der Eltern - und der lokalen Bevölkerung - lassen sich Innovationen kaum nachhaltig umsetzen. Besonders in kleinen Dörfern, wo die Schule eine zentrale Rolle einnimmt, hängt vieles vom Vertrauen und Verständnis der Eltern ab. Wie erwartet, zeigt sich ein gutes Schulklima im Einklang mit einer guten Elternpartizipation. Hier könnten Schulen eine gute Grundlage für die Vertrauensarbeit legen. Während unterrichtliche Massnahmen im Bereich der Differenzierung von den Eltern als unproblematisch angesehen werden, scheint AdL ein kontroverses Thema zu sein. Am wenigsten Probleme mit individualisiertem Unterricht haben die Eltern aus den Privatschulen, in welchen niveauund altersgemischter Unterricht die Regel ist. Da diese Schulen privat finanziert sind, ist anzunehmen, dass es sich eher um bildungsnahe Eltern handelt mit einem entsprechenden Einkommen. Die Meinungen der Eltern zum altersgemischten Unterricht variieren zwischen den Orten. Es zeigt sich eine grössere Zustimmung von Schulorten, welche im Projekt mitmachen und unter einem gewissen Druck stehen. Allerdings, und dies ist konsistent mit andern Befunden (Shumow, 2001), liegen die Meinungen innerhalb der Orte weiter auseinander als zwischen den Orten. Besonders hohe Zustimmung zeigt sich bei den Primarschulen. Die erste Oberstufe folgt an vierter Stelle und ist am weitesten fortgeschritten bei der Umsetzung von AdL. Sie weist gleichzeitig eine sehr hohe Elternsolidarität mit der lokalen Umsetzung des Projekts auf. Wenn der Druck von aussen (Schulschliessung) klein ist und die Eltern nicht zu einer Entscheidung drängt, dann könnte es für den Erfolg von Schulentwicklungsmassnahmen zweckmässiger sein, eine Versuchsphase vor der definitiven lokalpolitischen Entscheidung einzuschalten. Die Eltern hätten Zeit, sich konkret von den Vor- und Nachteilen überzeugen zu lassen und sich einzugewöhnen. Sie würden sehen, dass «die Kirche bzw. die Schule im Dorf bliebe». In den Ergebnissen hat sich gezeigt: Stimmt der Unterricht und lernen die Kinder gut, so sind die Eltern auch eher für eine "Altersdurchmischung» zu haben.

Insbesondere Eltern mit höherem Bildungsabschluss oder einer stärkeren Leistungsorientierung stehen Veränderungen im Bereich der Klassenzusammenlegung oder Unterrichtsgestaltung kritisch gegenüber. Dies ist auf dem Land, wo die Bevölkerung tendenziell konservativer eingestellt ist als in der Stadt, mit zu bedenken. Anliegen, die reformpädagogisch begründet werden, stossen auf Widerstand (Zbinden, 2011). Allerdings lässt sich gerade bei Reformschulen modellhaft gute Elternarbeit beobachten (Ullrich, 2008). Als Konsequenz ist darauf zu achten, alle Meinungen im Schulort aufzunehmen und Bedenken bei der weiteren Planung zu berücksichtigen. Eltern, die gewohnt sind, ihre Kinder zu Hause bei den Hausaufgaben kontrollierend zu unterstützen und die Angst haben, AdL sei mit Strukturverlust verbunden, müsste eine Weiterbildung zum Thema lernförderliche Hausaufgabenbetreuung angeboten werden. Gemeinsame Anliegen können die Eltern einen (Klaffke, 2000; Shumow, 2001). Ziel der 
Elterninformation wäre somit das Herausschälen eines gemeinsamen Interesses innerhalb der verschiedenen Meinungen der Eltern eines Schulortes. Der Erhalt der lokalen Schule unter Beachtung eines leistungsförderlichen Unterrichts könnte ein entsprechendes Ziel aus der Sicht der Eltern im Projekt Schule alpin sein.

Wild (2003) empfiehlt die Verbesserung der Elternarbeit durch Kooperation im Team. Dazu gehört auch, dass das Lehrpersonenteam sich zunächst einen eigenen Standpunkt, eine eigene Haltung zu einem Thema erarbeiten sollte. Hat das Team zu einer gemeinsamen pädagogischen Sicht gefunden, wird diese als Projektidee auch den Eltern vorgestellt. Entsprechend berichten die Eltern von einer besseren Elternpartizipation in unserer Untersuchung (siehe 6.4). Eltern können wichtige Beiträge leisten, ohne dass die «Profis» das Zepter aus der Hand geben müssen.

Grundsätzlich zeigt sich in der Untersuchung ein bedeutsamer Einfluss der Elternpartizipation auf das Schulentwicklungsprojekt. Dies äussert sich nicht nur in der direkten Unterstützung, sondern vor allem indirekt durch die Bedeutung der Elternpartizipation für die Zufriedenheit der Eltern mit dem bestehenden Unterricht und einer positiven Einstellung zur Altersdurchmischung, welche wiederum die Zustimmung zur Schulentwicklung positiv beeinflussen. Wenn Eltern auf Grund von positiven Erfahrungen schon in der Primarschule für einen differenzierenden, altersgemischten Unterricht gewonnen werden können, besteht bei einem Wechsel eines Kindes zur Oberstufe die Chance, dass die Zustimmung der Eltern auf der Oberstufe erhalten bleibt (Langer, 2011). Es besteht jedoch keine Garantie, dass Eltern ihre Meinung ändern, wenn Sie Erfahrungen mit Unterrichtsreformen gemacht haben (Shumow, 2001). Wird die Elternpartizipation von der reinen Konsultation ausgebaut zur Mitwirkung von einzelnen Eltern im Projekt, etwa in einer Steuergruppe, besteht aber eine erhöhte Chance, dass Eltern die Projektziele mittragen.

\section{Literaturverzeichnis}

Achermann, E. \& Gehrig, H. (2011). Altersdurchmischtes Lernen AdL. Bern: Schulverlag plus Aich, G. \& Behr, M. (2010). Kommunikation mit Eltern. In F. H. Müller, A. Eichenberger, M. Lüders \& J. Mayr (Hrsg.), Lehrerinnen und Lehrer lernen (S. 279-295). Münster: Waxmann.

Arnold, J., Kastner, H., Möckel, D., Otto, M., Weinberger, A. \& Wenzel, H. (2004). Schülerund Elternpartizipation in Schulentwicklungsprozessen. Halle: Landesinstitut für Lehrerfortbildung, Lehrerweiterbildung und Unterrichtsforschung von Sachsen-Anhalt.

Baumgartner, T., Lipowski, M. B. \& Rush, C. (2003). Increasing reading achievement of primary and middle school students through dfferentiated instruction. Unpublished Master of Arts Research Project, Saint Xavier University.

Bräu, K. (2008). Lehrerhandeln im Kontext Innerer Differenzierung. TriOS, 1, 21-34.

Bryk, A. S. \& Raudenbush, S. W. (1992). Hierarchical linear models. Thousands Oaks, CA: Sage Publications.

Christenson, S. \& Sheridan, S. M. (2001). Schools and families: Creating essential connections for learning. New York: Guilford Press. 
Cusin, C. \& Grossenbacher, S. (2001). Im Schnittpunkt der Veränderungen. Aarau: Schweizerische Koordinationsstelle für Bildungsforschung (SKBF).

Deci, E. L. \& Ryan, R. M. (1985). Intrinsic motivation and self-determination in human behavior. New York: Plenum Press.

Epstein, J. L. \& Dauber, S. L. (1991). School programs and teacher practices of parent involvement in inner-city elementary and middle schools. The Elementary School Journal, 91 (3), 289-305.

French, D., Atkinson, M. \& Rugen, L. (2007). Creating small schools: A handbook for raising equity and achievement. Thousand Oaks: Corwin Press.

Glauz, H. (2000). Beteiligung von Eltern an Schulentwicklungsprozessen. Organisationsberatung-Supervision-Clinical Management, 3, 225-236.

Griffith, J. (2000). School climate as group evaluation and group consensus: Student and parent perceptions of the elementary school environment. The Elementary School Journal, $101(1), 35-61$.

Hattie, J. A. C. (2002). Classroom composition and peer effects. International Journal of Educational Research, 37, 449-481.

Hoover-Dempey, K. V., Battiato, A. C., Walker, J. M. T., Reed, R. P., De Jong, J. M. \& Jones, K. P. (2001). Parental involvement in homework. Educational Psychologist, 36 (3), 195-209.

Hoover-Dempey, K. V. \& Sandler, H. M. (1997). Why do parents become involved in their children's education? Review of Educational Research, 67 (1), 3-42.

Hoover-Dempey, K. V., Walker, J. M. T., Sandler, H. M., Whetsel, D., Green, C. L., Wilkins, A. S. \& Closson, K. (2005). Why do parents become involved? Research findings and implications. The Elementary School Journal, 106 (2), 105-130.

Hox, J. (2002). Multilevel analysis. Mahwah: Lawrence Erlbaum.

Izzo, C. V., Weissberg, R. P., Kasprow, W. J. \& Fendrich, M. (1999). A longitudinal assessment of teacher perceptions of parent involvement in children's education and school performance. American Journal of Community Psychology, 7 (6), 817-839.

Keller, A. (2011). Begleitung und Unterstützung von kleinen Schulen. In R. Müller, A. Keller, U. Kerle, A. Raggl \& E. Steiner (Hrsg.), Schule im alpinen Raum (S. 417-434). Innsbruck: Studienverlag.

Klaffke, T. (2000). Eltern als Partner für Schulentwicklung. Lernende Schule, 10, 16-18.

Kliewe, A., Schreiber, D. \& Wiganek, G. (2006). Zusammenarbeit Elternhaus und Schule (k)eine Illusion. SchulVerwaltung spezial, 2, 21-23.

Langer, R. (2011). Schüler/innen, Eltern, externe Anspruchsgruppen. In H. U. Grunder, K. Kansteiner-Schänzlin \& H. Moser (Hrsg.), Schulentwicklung (S. 103-113). Hohengehren: Schneider.

Little, A. W. (2001). Multigrade teaching: Towards an international research and policy agenda. International Journal of Educational Development, 21, 481-497.

Lorenz, F. \& Wild, E. (2007). Parental involvement in schooling - results concerning its structure and impact on students' motivation. In M. Prenzel \& L. Allolio-Näcke (Hrsg.), Studies on the educational quality of schools. The final report on the DFG Priority Programme (pp. 299-316). Münster: Waxmann.

Lüdtke, O., Robitzsch, A., Trautwein, U. \& Köller, O. (2007). Umgang mit fehlenden Werten in der psychologischen Forschung. Probleme und Lösungen. Psychologische Rundschau, 58 (2), 103-117.

Meister, G. (2010). Partizipation von Eltern im Schulentwicklungsprozess. In T. Bohl, W. Helsper, H. G. Holtappels \& C. Schelle (Hrsg.), Handbuch Schulentwicklung. Theorie - Forschungsbefunde - Entwicklungsprozesse - Methodenrepertoire (S. 277-280). Bad Heilbrunn: Verlag Julius Klinkhardt.

Miller, B. A. (1991). A review of the qualitative research on multigrade instruction. Journal of Research in Rural Education, 7 (2), 3-12. 
Müller, R., Keller, A., Kerle, U., Raggl, A. \& Steiner, E. (Hrsg.). (2011). Schule im alpinen Raum. Innsbruck: Studienverlag.

Nägeli, A. (2011). Adaptive Schulmodelle am Beispiel innovativer Kleinschulen. In R. Müller, A. Keller, U. Kerle, A. Raggl \& E. Steiner (Hrsg.), Schule im alpinen Raum. Innsbruck: Studienverlag.

Shumow, L. (2001). Parents' educational beliefs: Implications for parent participation in school reforms. In S. Redding \& L. Thomas (Ed.), The community of the school. Lincoln, IL: Academic Development Institute.

Simon, B. S. (2004). High school outreach and family involvement. Social Psychology of Education, 7, 185-209.

Smith, E. P., Connell, C. M., Wright, G., Sizer, M., Norman, J. M., Hurley, A. \& Walker, S. N. (1997). An ecological model of home, school, and community partnerships: Implications for research and practice. Journal of Educational and Psychological Consultation, 8 (4), 339-360.

Smit, R., Humpert, W., Obertüfer-Gahler, R., Engeli, E. \& Breuer-Brodmüller, M. (2011). Differenzierung als Chance für kleine Schulen - Empirische Befunde im Längsschnitt. In R. Müller, A. Keller, U. Kerle, A. Raggl \& E. Steiner (Hrsg.), Schule im alpinen Raum. Innsbruck: Studienverlag.

Tieso, C. L. (2005). The effects of grouping practices and curricular adjustments on achievement. Journal for the Education of the Gifted, 29 (1), 60-89.

Tomlinson, C. A., Brighton, C., Hertberg, H., Callahan, C. M., Moon, T. R., Brimijoin, K., Conover, L. A. \& Reynolds, T. (2003). Differentiating instruction in response to student readiness, interest, and learning profile in academically diverse classrooms: A review of literature. Journal for the Education of the Gifted, 27 (2), 119-145.

Trautwein, U., Lüdtke, O., Schnyder, I. \& Niggli, A. (2006). Predicting homework effort: Support for a domain-specific, multilevel homework model. Journal of Educational Psychology, 98 (2), 438-456.

Ullrich, H. (2008). Ursprünglich für die Schwachen. Die Schulen der klassischen Reformpädagogik - was sie waren und was aus ihnen geworden ist. In W. Lohfeld (Hrsg.), Gute Schulen in schlechter Gesellschaft (S. 79-107). Wiesbaden: VS Verlag für Sozialwissenschaften.

Veenmann, S. (1995). Cognitive and noncognitive effects of multigrade and multi-age classes: A best-evidence synthesis. Review of Educational Research, 65 (4), 319-381.

Wild, E. (2003). Einbeziehung des Elternhauses durch Lehrer: Art, Ausmass und Bedingungen der Elternpartizipation aus der Sicht von Gymnasiallehrern. Zeitschrift für Pädagogik, 49 (4), 513-533.

Zbinden, H. (2011, 7. April). Dankbares Opfer. Die Zeit, (15/7.4.2011). Abgerufen von http://www.zeit.de/2011/15/CH-Schule/komplettansicht (25.3.12)

Schlagworte: Altersgemisches Lernen, innere Differenzierung, Schulentwicklung, ländliche Schulen, Eltern-Partizipation 


\section{Le regard de parents à propos d'une expérience d'enseignement dans des classes à degrés multiples (projet "Schule alpin», part. St. Gall)}

\section{Résumé}

Les parents sont des partenaires importants dans le cadre des projets de développement scolaires, d'autant plus si des mesures de différenciation pédagogiques proposant un enseignement multi-âges dans des classes à degrés multiples sont introduites. L'introduction de ces classes a pour objectif de sauvegarder les «petites écoles» des Alpes qui accueillent des élèves issus de milieux hétérogènes. Suite à la passation d'un questionnaire, le rapport qu'entretiennent 682 parents de 19 écoles différentes avec ce type de dispositif a été étudié. L'analyse des données a été réalisée selon les règles de la statistique descriptive en s'appuyant entre autres sur la comparaison des moyennes, sur la modélisation d'équations structurelles et l'analyse multi-niveaux. Il s'est avéré que les parents les moins centrés sur la performance et les plus satisfaits des occasions de participation parentale qui leur sont offertes approuvent clairement l'enseignement multi-âges et le projet de développement scolaire dont il est issu. En conclusion, l'article discute la pertinence de ces résultats pour le développement futur des «petites écoles» dans les zones alpines.

Mots clés: Enseignement multi-âges, différenciation pédagogique, développement scolaire, écoles rurales, participation parentale

\section{Il punto di vista dei genitori a proposito dell'insegnamento sotto forma di classi di età mista nell'ambito del progetto di sviluppo scolastico "Schule alpin" di San Gallo}

\section{Riassunto}

Nell'ambito di progetti per lo sviluppo della scuola i genitori rappresentano delle controparti importanti, soprattutto se si ha come obiettivo quello di mantenere piccole sedi scolastiche alpine, con classi eterogeneeorganizzate sotto forma di classi di età mista con una corrispondente differenziazione interna. 682 genitori di 19 scuole sono stati contattati con un questionario per sondare il loro atteggiamento nei confronti di vari aspetti legati all'organizzazione scolastica menzionata

L'analisi dei dati (statistica inferenziale) è stata svolta ricorrendo alla comparazione dei valori medi, ai modelli di analisi del cammino e alle analisi multilevel. Tra le altre cose si è visto come i genitori caratterizzati da valori più bassi nell'orientamento verso le prestazioni e da una soddisfazione maggiore per la partecipazione dei genitori mostrano un grado maggiore di approvazione nei confronti della didattica per classi di età eterogenee e del progetto di sviluppo 
della scuola. Viene discusso quale importanza possono avere i risultati per uno sviluppo ulteriore delle scuole nelle zone alpine e rurali.

Parole chiave: Apprendimento per classi di età eterogenee, differenziazione interna, sviluppo della scuola, scuole in aree rurali, partecipazione dei genitori

\section{Parents' perspective on multi-age teaching within a school development initiative (project "schools in alpine regions", St. Gall part)}

\section{Summary}

Parents are important partners in school development projects, in particular if the aim of the project is to save small schools from getting shut down by introducing multi-age classes and differentiated instruction in their children's classes. On the basis of a questionnaire, 682 parents of 19 different school locations were asked what they thought about these reforms and this specific form of education.

The data was analysed by comparison of means, path and multilevel analysis. Results show that parents with lower children's achievement expectation and with higher satisfaction concerning parent participation agree in general more with multi-age teaching and the development project itself. The relevance of the findings for the further development of rural-alpine schools is discussed.

Keywords: Multi-age learning, differentiated instruction, school improvement, rural schools, parent participation 